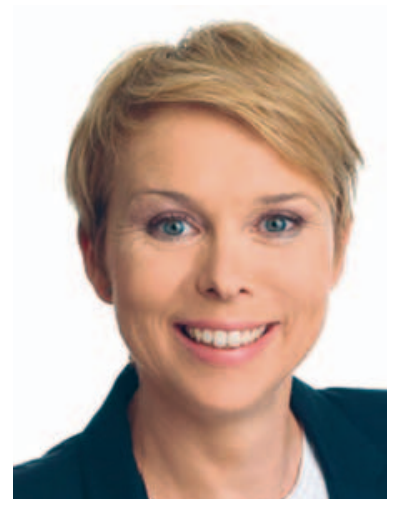

Christine Keller Sallenbach, Bern, Schweiz

\section{Komplementärmedizin 5 Jahre nach der Abstimmung}

Am 17. Mai 2009 nahm das Schweizer Volk mit einer Zweidrittelmehrheit die Vorlage «Ja zur Komplementärmedizin» an und verankerte den Artikel 118a Komplementärmedizin in der Verfassung: «Bund und Kantone sorgen im Rahmen ihrer Zuständigkeiten für die Berücksichtigung der Komplementärmedizin.» Ein für alle Mal wurde klar, wie breit die Komplementärmedizin in der Bevölkerung abgestützt ist.

Mit dem Verfassungsartikel 118a Komplementärmedizin sind folgende fünf Kernforderungen verbunden:

- Förderung der integrativen Medizin (Zusammenarbeit von Schul- und Komplementärmedizin);

- Aufnahme ärztlicher Richtungen der Komplementärmedizin in die Grundversicherung und in die weiteren Sozialversicherungen (SUVA, Militär- und Invalidenversicherung);

- Förderung von Lehre und Forschung;

- Schaffung nationaler Diplome und kantonaler Berufszulassungen für nichtärztliche Therapeuten;

- Sicherstellung der Heilmittelvielfalt.

Wo steht die Komplementärmedizin heute? Von diesen Kernforderungen ist bis heute keine vollständig umgesetzt (siehe auch Beitrag auf Seite 268), und ohne den ständigen Druck des Dachverbandes Komplementärmedizin (Dakomed) auf Politik, Behörden und Verwaltung sähe die Bilanz wohl deutlich schlechter aus.
Der Dakomed ist der kleine Stachel im Fleisch von Politik und Verwaltung. Er trägt als Bürgerbewegung und Branchenorganisation den Abstimmungserfolg weiter. Er setzt sich für die Umsetzung des Volkswillens ein, indem er Politik, Behörden und Verwaltung auf die Finger schaut und konkrete Vorschläge ausarbeitet.

In den letzten 5 Jahren hat sich der Dakomed hauptsächlich auf Bundesebene für die Komplementärmedizin eingesetzt. Wichtige Gesetzesrevisionen für die Komplementärmedizin stehen vor dem Abschluss. Das Heilmittelgesetz, welches eine vereinfachte Zulassung für komplementärmedizinische und Phytoarzneimittel vorsieht, wurde bereits vom Nationalrat beraten. Lesen Sie dazu das Interview mit Yvonne Gilli auf Seite 259. Das Medizinalberufegesetz, welches in den Ausbildungszielen von Medizinern und Pharmazeuten Grundkenntnisse über Komplementärmedizin vorsieht, wird wohl im Herbst 2014 vom Zweitrat verabschiedet, und die definitive Aufnahme der ärztlichen Komplementärmedizin in die Grundversicherung wurde vom Bundesrat angekündigt und wartet jetzt auf die Umsetzung (siehe Beitrag hierzu auf Seite 264). Alle diese Erfolge dürfen aber nicht darüber hinwegtäuschen, dass es weiterhin eine starke Interessenvertretung für die Komplementärmedizin braucht. Denn ohne ständigen Druck auf Politik und Behörden gingen die Anliegen der Komplementärmedizin im heutigen Politikbetrieb glatt vergessen. Die parteiübergreifende parlamentarische Gruppe Komplementärmedizin, in welcher sich 15 Ständeräte und Ständerätinnen sowie 44 Nationalräte und Nationalrätinnen zusammengefunden haben, ist eine wichtige Stütze für die Umsetzung der Anliegen der Komplementärmedizin im Parlament.

Der Fokus des Dakomed wird in den nächsten Jahren stärker bei den Kantonen liegen, denn diese sind mehrheitlich für das Gesundheitswesen zuständig. Ihnen obliegt die Spitalplanung, ebenso die Universitäten und die Berufszulassung von Ärzten, Naturheilpraktikern und nichtärztlichen Therapeuten.

Diese Arbeit kann der Dakomed nur dank der finanziellen Unterstützung von vielen tausend Kleinspendern, unseren Mitgliederorganisationen und unseren Gönnern leisten. Diese Spendenbereitschaft zeigt auch die starke Verankerung der Komplementärmedizin in der Bevölkerung (siehe auch Artikel auf Seite 274). Dies sind die Voraussetzungen dafür, dass der Dakomed seine politische Arbeit mit Erfolg weiterführen kann.

\section{KARGER}

Fax +497614520714 Information@Karger.com www.karger.com
Christine Keller Sallenbach

Geschäftsführerin Dachverband Komplementärmedizin (Dakomed)

Amthausgasse 18, 3011 Bern, Schweiz

christine.keller@dakomed.ch

www.dakomed.ch 